\title{
A Note on Moduli Stabilisation in Heterotic Models in the Presence of Matter Fields
}

\author{
Andrei Micu \\ Department of Theoretical Physics \\ National Institute for Physics and Nuclear Engineering - Horia Hulubei \\ Str. Atomiştilor 407, P.O. Box MG-6 Măgurele, Romania \\ amicu@th.physik.uni-bonn.de
}

\begin{abstract}
In this brief note we analyse a toy model which can be derived from heterotic string compactifications on half-flat manifolds with $S U(3)$ structure at first order in $\alpha^{\prime}$ (ie including matter fields). We show that for this model, finding solutions with viable gauge group poses the same problems as finding solutions in the absence of matter fields.
\end{abstract}

December 2008 


\section{Introduction}

Moduli stabilisation in the presence of background fluxes has been an active field of research in the last years. In particular, several solutions with stabilised closed string moduli were found. Most cases include type IIB string compactifications, but examples within type IIA or heterotic strings as well as M-theory have also been constructed. By now one can say that there is a fair understanding of moduli stabilisation in such models.

On the other hand, this problem has been addressed separately from the issue of constructing a Standard Model-like sector. Several string models which reproduce the Standard Model spectrum and gauge group are known, but moduli stabilisation in these scenarios is still an open question. It has been largely assumed (at least in type IIB scenarios) that the two sectors are independent and one can always add the Standard Model sector after the closed string moduli have been stabilised. This procedure was questioned in ref. [1] where it was shown that certain constraints have to be fulfilled in order to avoid the destabilisation of the moduli vacuum by the matter sector. Few examples have been analysed in the context of type IIB compactifications in refs. [2, 3]. However, the interaction between these two sectors still needs a better understanding and therefore, it is important to address the problem of the moduli stabilisation in realistic models.

The present note represents a small step in this direction in that we try to analyse a simple model and see what are the obstacles against a viable solution with all moduli stabilised in the presence of matter fields. The model we shall discuss can be obtained from heterotic string compactifications. The appearance of a visible sector is almost automatic in such scenarios and therefore the only thing we have to worry about is actually moduli stabilisation. This is not an easy task as in heterotic string compactifications the only ordinary flux available is the NS-NS $H$-flux. Hence, we are forced to take into account also geometric fluxes ie compactifications on manifolds with torsion. Here we shall use the models derived in refs. [4, 5, 6] where so called half-flat manifolds with $S U(3)$ structure and generalisations thereof were considered. Such models have a $E_{6}$ gauge group, chiral matter transforming under $\mathbf{2 7}$ and $\overline{\mathbf{2 7}}$ and singlet fields coming from the geometric moduli of the compactification manifold. We will show that, a simple setup which contains only Kähler moduli and charged fields transforming as $\overline{\mathbf{2 7}}$ under the $E_{6}$ gauge group, does not have satisfactory supersymmetric solutions. In particular, we will see that the problems encountered in finding reliable solutions can be related to the fact that in the absence of matter fields such systems do not have satisfactory solutions either.

\section{The model}

As explained in the Introduction, the model we deal with can be derived from compactifications of the heterotic string on half-flat manifolds with $S U(3)$ structure [4, 5]. We will consider a simplifying case in that we take $h^{2,1}=0$, where $h^{2,1}$ stands for the corresponding Hodge number of the original Calabi-Yau manifold (in other words these

manifolds correspond to rigid Calabi-Yau's and have no complex structure moduli). The 
model will therefore be $N=1$ supergravity coupled to a super Yang-Mills sector with gauge group $E_{6}$ with a certain number $h$ of charged fields $C^{i}, i=1 \ldots h$ transforming as $\overline{\mathbf{2 7}}$ of $E_{6}$ and $h$ Kähler moduli $T^{i}$ which are singlets under the gauge group 11 In principle one also has to take into account the dilaton field, but it will play no role in the present discussion and we will therefore ignore it for the sake of simplicity. The field $C^{i}$ carries an additional index $A$ running over the 27-dimensional representation of $E_{6}$. In the following we shall suppress the index $i$ and effectively work with $h=1$, but the results can be straightforwardly generalised to the case $h>1$.

Before we proceed with the model we should note that the case $h^{2,1}=0$ may not be totally irrelevant from the string compactification point of view. Suppose we consider a compactification on a Calabi-Yau manifold with $h^{1,1}>h^{2,1} \neq 0$. This model will include also complex structure moduli, denoted by $Z$, as well as charged fields transforming under 27 of $E_{6}$, which we denote by $D$. In ref. [5] it was shown that the geometric fluxes can give mass couplings to the fields $(T, Z)$ and $(C, D)$. By choosing the flux parameters appropriately we can give large masses to $h^{2,1}$ pairs of moduli fields $(T, Z)$ as well as to the corresponding charged fields $(C, D)$, and integrating them out, we are left precisely with the field content of the model we want to discuss in this note.

Coming back to the model we want to analyse, the Kähler potential for the chiral fields is given by [5]

$$
K=-3 \log (T+\bar{T})+\alpha^{\prime} \frac{6}{(T+\bar{T})} C^{A} \bar{C}_{A}+\mathcal{O}\left(\alpha^{\prime 2}\right)
$$

and the superpotential

$$
W=i e T-\frac{\alpha^{\prime}}{3} j_{A B C} C^{A} C^{B} C^{C}+\mathcal{O}\left(\alpha^{\prime 2}\right)
$$

The two pieces in this superpotential are not new, but were never studied simultaneously before. In particular, the cubic term has been known since the mid eighties, [8], but at that time the moduli superpotential coming from fluxes (including geometrical ones) was not developed. The linear piece in the superpotential was studied in ref. [7] and it has been concluded that this piece alone can not solve the stabilisation problem for the Kähler modulus $T$. The main question we want to ask in this note is whether the addition of the cubic term in the superpotential changes the conclusions of ref. [7]. As we shall see in the following the problems we encounter in stabilising the moduli in this model can be traced back to the problems encountered in ref. [7].

\footnotetext{
${ }^{1}$ We ignore the bundle moduli in this analysis.

${ }^{2}$ The factors which appear in these formulae can be derived from [5], but their precise values are not relevant in the following.
} 


\section{Solutions}

The supersymmetric solutions of this system can be found by solving the F-term equations

$$
\begin{aligned}
F_{T} & =i e-\frac{3}{T+\bar{T}} W-\frac{6 \alpha^{\prime} C^{A} \bar{C}_{A}}{(T+\bar{T})^{2}} W+\mathcal{O}\left(\alpha^{\prime 2}\right) \\
F_{C^{A}} & =\alpha^{\prime}\left(-j_{A B C} C^{B} C^{C}+\frac{6 \bar{C}_{A}}{(T+\bar{T})} W\right)+\mathcal{O}\left(\alpha^{\prime 2}\right) .
\end{aligned}
$$

The second equation is in general difficult to solve. However, we are not interested in the most general solution, but we are also looking for solutions which would be of relevance for the four-dimensional physics, ie solutions which may include sectors of the standard model. Therefore it would be interesting to see if there exist solutions which preserve a gauge group which is large enough to incorporate the standard model gauge group. Possible solutions within $E_{6}$ are: $E_{6}$ itself, $S O(10) \times U(1), S U(3) \times S U(3) \times S U(3)$ or $S U(2) \times S U(6)$. Under these subgroups $\overline{\mathbf{2 7}}$ branches as [9]

$$
\begin{aligned}
& \overline{\mathbf{2 7}}=\overline{\mathbf{1 6}}^{1} \oplus \mathbf{1 0}^{-2} \oplus \mathbf{1}^{4} ; \\
& \overline{\mathbf{2 7}}=(\mathbf{3}, \overline{\mathbf{3}}, \mathbf{1}) \oplus(\overline{\mathbf{3}}, \mathbf{1}, \overline{\mathbf{3}}) \oplus(\mathbf{1}, \mathbf{3}, \mathbf{3}) ; \\
& \overline{\mathbf{2 7}}=(\overline{\mathbf{2}}, \mathbf{6}) \oplus(\mathbf{1}, \mathbf{1 5}) .
\end{aligned}
$$

By giving a vev to the singlet in eq. (3.3), $E_{6}$ is broken to $S O(10)$, which from a phenomenological point of view, is one of the most interesting GUT choices. A vev for any of the fields on the RHS of eq. (3.4) breaks the gauge group to $S U(3) \times S U(2) \times S U(2)$. Finally, a vev for the $(\overline{\mathbf{2}}, \mathbf{6})$ breaks the gauge group to $U(1) \times S U(5)$ while a vev for $(\mathbf{1}, \mathbf{1 5})$ breaks the gauge group to $S U(2) \times S U(4) \times S U(2)$. We shall analyse these possibilities in the following subsections.

\section{$3.1 \quad E_{6}$ preserving solution}

A solution which preserves the $E_{6}$ gauge group necessarily has vanishing charged fields, ie $<C^{A}>=0$. Then equation (3.2) is automatically satisfied and we are left with eq. (3.1). This is a simplified version of the equations which were analysed in ref. [7] and has no solution for non-vanishing flux parameter $e$.

Note that there is a possible way around this problem. This however is not in the main line of this note as it has vanishing matter fields and, therefore, could have been studied before, but for the sake of completeness we show why it does not represent a viable solution either. As we explained in the introduction, the model we consider here is definitely not a complete one and it can actually be considered to emerge after integrating out massive fields at a higher energy scale. In this case one would also have to consider an additional constant, $w^{0}$, in the superpotential which represents the value of the superpotential at the point where the integrated out fields were stabilised. There is no reason to believe that this constant is real and therefore equation (3.1) will have 
the solution

$$
T=-\frac{1}{e}\left(3 \operatorname{Im} w^{0}-i R e w^{0}\right) .
$$

Since the flux parameter $e$ is expected to be quantised [7], in order to obtain a trustworthy solution for the Kähler modulus we need a large $\operatorname{Im} w^{0}$. On the other hand we have to remember that the full model also contains the dilaton which has to be stabilised as well. The only known solution is through gaugino condensate in the hidden sector which can give a large value for the dilaton field when $W$ is small. Therefore it seems impossible to stabilise both the Kähler moduli and the dilaton at large values in this way.

In conclusion, as expected, $E_{6}$ preserving solutions are in no respect different from the solutions found in ref. [7].

\section{$3.2 S O(10)$ preserving solutions}

One of the phenomenologically most promising subgroups of $E_{6}$ is $S O(10)$. The branching of the 27-dimensional representation of $E_{6}$ under $S O(10) \times U(1)$ is given in eq. (3.3) where the superscripts indicate the $U(1)$ charge. Clearly, giving a vev to the singlet in eq. (3.3) breaks the gauge group from $E_{6}$ down to $S O(10)$. Let us also split the charged field $C^{A}$ according to the above branching

$$
C^{A}=\left(C^{\alpha}, C^{a}, c\right)
$$

where $\alpha$ and $a$ run over the $\overline{\mathbf{1 6}}$ and $\mathbf{1 0}$ dimensional representations of $S O(10)$ respectively. By $c$ we have denoted the singlet from eq. (3.3) and therefore we are looking for a situation where

$$
<C^{\alpha}>=<C^{a}>=0, \quad<c>\neq 0 .
$$

Since the singlet $c$ is is charged under $U(1)$ a coupling of the type $c^{3}$ is forbidden and therefore the only allowed couplings have the generic form $\overline{\mathbf{1 6}} \cdot \overline{\mathbf{1 6}} \cdot \mathbf{1 0}$ and $\mathbf{1 0} \cdot \mathbf{1 0} \cdot \mathbf{1}$.

Writing eq. (3.2) for the component field $c$ we obtain

$$
F_{c}=-\alpha^{\prime} j_{a b 1} C^{a} C^{b}+K_{c} W=0 .
$$

The first term vanishes due to eq. (3.8) and since $K_{c} \sim \bar{c} /(T+\bar{T})$ can only vanish in the decompactification limit $T+\bar{T} \rightarrow \infty$, we conclude that $W$ must vanish for such a solution

$$
W=0
$$

Clearly, for non-vanishing flux parameter e, equation (3.1) does not have a solution which implies that supersymmetric $S O(10)$ solutions do not exist in this toy model. As anticipated, this problem can be traced back to the problems encountered in ref. [7]. In particular we see that the absence of supersymmetric solutions at the zeroth order in $\alpha^{\prime}$ prevents the existence of solutions also at the first order in $\alpha^{\prime}$.

Note that one can think of breaking the gauge group further to $S U(5)$. This can be done by giving a vev to the singlet in the branching of $\overline{\mathbf{1 6}}$ under $S U(5)$. However, since 
the couplings $\overline{\mathbf{1 6}}^{3}$ or $\overline{\mathbf{1 6}}^{2} \cdot \mathbf{1}$ do not exist either, the above arguments remain unchanged even for breaking the gauge group to $S U(5)$. Therefore, the phenomenologically most interesting cases ie $S O(10)$ and $S U(5)$ gauge groups are ruled out in this simple model. It would be interesting to see if in more complicated models, with more complicated moduli and matter fields superpotentials, this conclusion may be changed. It is worth noting that if the condition $W=0$ persists this is still not satisfactory at least from the point of view of stabilising the dialton for which we need a small, but non-zero value for the superpotential.

\section{3 $S U(3) \times S U(2) \times S U(2)$ preserving solution}

This solution is intrinsically different from the $S O(10)$ solution. The reason is that the field which breaks $E_{6}$, say $(\mathbf{1}, \mathbf{3}, \mathbf{3})$, admits a coupling of the type $(\mathbf{1}, \mathbf{3}, \mathbf{3})^{3}$. Let's denote this field generically by $B^{a}$. Then the corresponding F-term equation will be of the form

$$
F_{B^{a}}=\alpha^{\prime}\left(j_{a b c} B^{b} \cdot B^{c}+\frac{6 \bar{B}_{a}}{T+\bar{T}} W\right) .
$$

For a non-vanishing $B$ we necessarily have $W \neq 0$. Note that this equation is an equation at the first order in $\alpha^{\prime}$ and therefore $W$ appearing on the RHS of it is only the flux superpotential term $e T$. Moreover, the approximation in which the starting theory can be trusted is one where the charged fields represent small fluctuations around zero. Therefore in the above equation $B$ has to be taken small and thus

$$
B \sim \frac{6 W}{T+\bar{T}}=\frac{6 i e T}{T+\bar{T}} \ll 1 .
$$

Since the fluxes are quantised this expression can not be made arbitrarily small. Moreover since the units in which the fluxes are quantised are of order $1 / \sqrt{\alpha^{\prime}}$, this solution can not be trusted as the $\alpha^{\prime}$ expansion we started with in eqs. (2.1) and (2.2) no longer makes sense. At a first glance it may seem that this problem is not related to the ones encountered in ref. [7] when looking for solutions. However, from (3.12) we see that actually the obstacle in this case is that we can not make $W$ arbitrarily small while keeping the Kähler modulus $T$ large. This was essentially the major obstruction in ref. [7] and was shown that more complicated moduli superpotentials may alleviate the problem.

\subsection{Other solutions}

The other possible breaking of $E_{6}$ which we have presented at the beginning of this section fall in one of the classes discussed above and we shall only briefly discuss them. In the case of breaking by the vev of $(\overline{\mathbf{2}}, \mathbf{6})$ in eq. (3.5), a coupling of the type $(\overline{\mathbf{2}}, \mathbf{6})^{3}$ does not exist and therefore, like in the $S O(10)$ case, the F-term equation for the field which acquires a vev imposes that $W=0$. As we discussed before this can not be a solution to eq. (3.1) for non-vanishing flux parameter $e$. The other case where the breaking of $E_{6}$ is due to a vev of $(\mathbf{1}, \mathbf{1 5})$ a cubic coupling for this field exists and therefore one can find a solution to the system (3.1) and (3.2), but as noted in the previous subsection such a solution is not consistent with all the approximations used in deriving this model. 


\section{Conclusions}

In this note we have studied a simple toy model of $N=1$ supergravity coupled to a visible sector with a gauge group $E_{6}$ with one chiral field in the $\overline{\mathbf{2 7}}$ representation of $E_{6}$ and a singlet field. Such models can be obtained from heterotic string compactifications on manifolds with $S U(3)$ structure (in particular half-flat manifolds) as described in ref. [5]. We have analysed the vacua of this model which have a chance to describe the Standard Model. The case when the gauge group $E_{6}$ is preserved is trivial in the sense that these solutions were discussed before in ref. [7]. For the cases where the group $E_{6}$ is broken we have encountered two situations

1. The field which acquires a vev has a cubic coupling in the superpotential. In this case, because the charged fields are considered to be small fluctuations around zero, the value for the superpotential at the critical point is required to be also small which is in tension with the fact that the value for the modulus field $T$ has to be large in order to be in a valid regime of the supergravity approximation we have been using. We found that this is the case for the solutions which break the gauge group to $S U(3) \times S U(2)^{2}$ or $S U(4) \times S U(2)^{2}$.

2. The field which acquires a vev does not have a cubic coupling in the superpotential. In this case there is simply no supersymmetric solution as the F-term equation for the field which gets a vev implies a vanishing value for the superpotential which is not compatible with the $F_{T}$ equation (3.1). We found that breaking to $S O(10)$ or $S U(5) \times U(1)$ lie in this class.

An important feature of the analysis in this note is that the fact that no satisfactory solutions in the presence of charged fields were found can be directly related to the fact that, for this model, in the absence of charged fields, no proper solutions exit, [7]. Therefore it is expected that in more general and more realistic models which also include complex structure moduli and which have proper solutions at the zeroth order in $\alpha^{\prime}$, [7], one can also find trustworthy solutions in the presence of charged fields. In particular, there will always be $E_{6}$ preserving solutions which are merely extensions of the solutions already obtained in ref. [7]. It is also expected that the solutions which fall in the first class above are better behaved in more complicated models as it was shown in ref. [7] that the moduli can be stabilised at reasonable values while keeping the superpotential small. It is not clear what will really happen with the solutions which fall in the second class above. If $W$ is still required to vanish there will be a problem in finding solutions for the dilaton from gaugino condensation in the hidden sector. If however, the conclusion $<W>=0$ can be avoided this will constitute a completely new case which will have to be analysed in detail. The analysis of these more complicated models is left for future work [10].

Acknowledgments The author thanks Shanta de Alwis for carefully reading a previous version of this manuscript. This work was supported by the National University Research Council (CNCSIS) under contract UEFISCSU 3/3.11.2008. 


\section{References}

[1] R. Blumenhagen, S. Moster and E. Plauschinn, "Moduli Stabilisation versus Chirality for MSSM like Type IIB Orientifolds," JHEP 0801 (2008) 058 arXiv:0711.3389 [hep-th]].

[2] R. Blumenhagen, S. Moster and E. Plauschinn, "String GUT Scenarios with Stabilised Moduli," arXiv:0806.2667 [hep-th].

[3] J. P. Conlon, A. Maharana and F. Quevedo, "Towards Realistic String Vacua," arXiv:0810.5660 [hep-th].

[4] S. Gurrieri, A. Lukas and A. Micu, "Heterotic String Compactified on Half-flat manifolds," Phys. Rev. D 70 (2004) 126009 |arXiv:hep-th/0408121.

[5] S. Gurrieri, A. Lukas and A. Micu, "Heterotic String Compactifications on Half-flat Manifolds II," JHEP 0712 (2007) 081 [arXiv:0709.1932 [hep-th]].

[6] T. Ali and G. B. Cleaver, "A Note on the Standard Embedding on Half-Flat Manifolds," JHEP 0807 (2008) 121 [arXiv:0711.3248 [hep-th]].

[7] B. de Carlos, S. Gurrieri, A. Lukas and A. Micu, "Moduli stabilisation in heterotic string compactifications," JHEP 0603 (2006) 005 arXiv:hep-th/0507173.

[8] E. Witten, "Dimensional Reduction Of Superstring Models," Phys. Lett. B 155 (1985) 151.

[9] R. Slansky, "Group Theory For Unified Model Building," Phys. Rept. 79 (1981) 1.

[10] A. Micu, "Moduli stabilisation in heterotic GUT models", work in progress 